JOURNAL OF

APPLIED

CRYSTALLOGRAPHY

ISSN 1600-5767

\section{High-resolution SAXS setup with tuneable resolution in direct and reciprocal space: a new tool to study ordered nanostructures}

\author{
Andrei P. Chumakov, ${ }^{\text {a* }}$ Kirill S. Napolskii, ${ }^{\text {b }}$ Andrei V. Petukhov, ${ }^{\text {,d }}$ Anatoly A. \\ Snigirev, ${ }^{\mathrm{e}}$ Irina I. Snigireva, ${ }^{\mathrm{a}}$ Ilya V. Roslyakov ${ }^{\mathrm{b}}$ and Sergey V. Grigoriev ${ }^{\mathrm{f}, \mathrm{g}}$
}

Received 12 May 2019

Accepted 11 August 2019

Edited by S. Boutet, SLAC National Accelerator Laboratory, Menlo Park, USA

Keywords: compound refractive lenses; smallangle X-ray scattering; microradian diffraction; anodic alumina.

Supporting information: this article has supporting information at journals.iucr.org/j

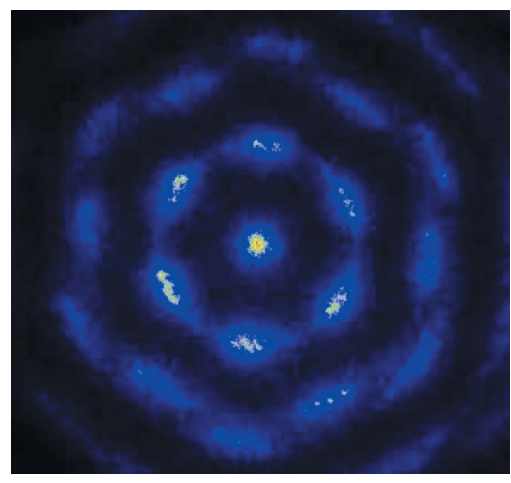

C 2019 International Union of Crystallography

\begin{abstract}
aEuropean Synchrotron Radiation Facility, 71 Avenue des Martyrs/BP 220, Grenoble 38043, France, ${ }^{\mathbf{b}}$ Lomonosov Moscow State University, Moscow 119991, Russian Federation, 'van 't Hoff laboratory for Physical and Colloid Chemistry, Debye Institute for Nanomaterials Science, Utrecht University, Padualaan 8, Utrecht 3508, The Netherlands, dLaboratory of Physical Chemistry, Department of Chemical Engineering and Chemistry, Eindhoven University of Technology, The Netherlands, $\mathbf{e}$ Immanuel Kant Baltic Federal University, Nevskogo Street 14, Kaliningrad 236041, Russian Federation, 'Physics Institute, NRC Kurchatov Institute, Gatchina, Leningrad Region 188300, Russian Federation, and ${ }^{\mathbf{g}}$ Faculty of Physics, Saint-Petersburg State University, Saint-Petersburg, 198504, Russian Federation. *Correspondence e-mail: a.p.chumakov@gmail.com
\end{abstract}

A novel compact small-angle X-ray scattering (SAXS) setup with tuneable resolution in both direct and reciprocal space has been designed and tested for the study of nanostructured materials with a hierarchical structure. The setup exploits a set of compound refractive lenses that focus the X-ray beam at the detector position. Anodic alumina membranes with a self-ordered porous structure were chosen as test samples. The setup allows patterns to be collected with a minimum scattering vector value of $0.001 \mathrm{~nm}^{-1}$ and gives the possibility for an easy continuous switch between taking high-resolution statistically averaged diffraction data of macroscopically large sample volumes and lowerresolution diffraction on a small single domain of the anodic aluminium oxide film. It is revealed that the pores are longitudinal and their ordering within each domain tends towards the ideal hexagonal structure, whereas the in-plane orientation of the pore arrays changes from domain to domain. The possible advantages and disadvantages of the proposed compact SAXS scheme are discussed.

\section{Introduction}

For precise structural characterization, microscopy and diffraction methods are complementary and provide information about the local and average structure of the material in direct and reciprocal space, respectively. Similar to X-ray diffraction which is usually applied for the identification and refinement of crystal structures, small-angle X-ray scattering (SAXS) is used for the analysis of materials that possess ordering of their structure on the nano- or microscale. SAXS setups with high resolution mainly utilize synchrotron radiation. Recording the scattering at small angles assumes the presence of such structural features of the SAXS installation as (i) a long collimating section from the source of the synchrotron beam to the sample (several tens of metres), (ii) a fixed sample position and (iii) a considerable distance from the sample to the detector (detector tube), which can reach 10-30 m (Narayanan et al., 2018; Smith et al., 2019; Nikitenko et al., 2008). Alternatively, one can use a more compact Bonse-Hart camera (Ilavsky et al., 2009), although this is not useful for recording 2D scattering patterns. The standard procedure for forming a synchrotron beam and the fixed 
position of the sample stage in most cases make it impossible to vary the illuminated sample area without realigning the SAXS setup. The exception is the possibility of changing the illuminated area of the sample by changing the beam-defining slits upstream of the sample position in primary or secondary short-focusing schemes. However, the change in beam size in this case will lead to a rapid reduction in the intensity, especially if the size of the desired illuminated area is of the order of several micrometres. A large sample-to-detector distance requires the availability of a long accessible physical space, and large financial and time costs (design, manufacture, complexity in the maintenance of mechanical and vacuum systems, etc.). The problem of separating the weak small-angle scattered intensity from the intense divergent direct beam creates additional difficulties. Most of these problems could be overcome by focusing the beam.

The discovery of a new method of focusing X-ray beams and the invention of compound refractive lenses (CRL) for X-ray optics (Snigirev et al., 1996; Lengeler et al., 2005) allowed a breakthrough in the development of new synchrotron setups and facilities (Vaughan et al., 2011; Seiboth et al., 2014). In particular, CRL can modify and simplify SAXS setups (Petukhov et al., 2006, 2015) and create various facilities for the study of the local microstructure of matter by diffraction methods (Snigireva \& Snigirev, 2006; Riekel et al., 2010). The combination of standard and novel approaches in instrument design leads to new experimental opportunities and data-collection technologies (Bosak et al., 2010; Byelov et al., 2013, Santoro et al., 2014).

Here we introduce a novel high-resolution SAXS setup with tuneable resolution in both direct and reciprocal space for the study of nanostructured materials. It is especially suited for the characterization of nanomaterials possessing a hierarchical structure with simultaneous ordering on the nano- and microscales. The performance capabilities of the suggested scheme were tested using porous anodic aluminium oxide (AAO) films, the structure of which consists of micrometresized domains with a 2D hexagonal array of sub- $100 \mathrm{~nm}$ sized cylindrical channels. There is also long-range in-plane orientational order in the AAO structure extending over the millimetre scale (Napolskii et al., 2012). The advantages and disadvantages of this novel SAXS scheme with tuneable resolution in direct and reciprocal space are discussed.

\section{Description of the novel SAXS scheme}

\subsection{Microradian SAXS and X-ray beam coherence}

Since a scattering pattern is created as a result of the interference of waves scattered at the sample, beam coherence is a crucial issue for high-resolution scattering experiments. One usually defines the longitudinal coherence $l_{\text {long }}$, which is related to the final spectral width $\Delta \lambda$ of the $\mathrm{X}$-rays, and the transverse coherence $l_{\text {tr }}$, which depends on the final size of the radiation source. Under the conditions of most small-angle $\mathrm{X}$-ray scattering experiments exploiting a silicon monochromator (typically, $\Delta \lambda / \lambda \simeq 10^{-4}$ ), the longitudinal coher- ence $l_{\text {long }}=\lambda^{2} /(2 \Delta \lambda)$ is sufficient to provide interference between waves scattered at different depths in the sample (Petukhov et al., 2002; Grigoriev et al., 2010; Als-Nielsen \& McMorrow, 2011).

It is more challenging to ensure sufficient beam coherence in the transverse direction. Owing to the finite source size $S$, the beam consists of coherent volumes (or patches) that are incoherent with each other. For a freely propagating beam, the size of a coherent patch is determined by the transverse coherence length, which increases linearly with increasing distance (Born \& Wolf, 1970; Pauw, 2013). For example, at the position of the CRL it is given by

$$
l_{\mathrm{tr}, 0}=\frac{R \lambda}{2 S},
$$

where $R$ is the distance travelled by the beam from the source to the CRL and $\lambda$ is the wavelength. The angular resolution of a SAXS scheme determines the maximum scale at which electron-density correlations can be determined. By using values typical for synchrotron facilities of $R \simeq 50 \mathrm{~m}, \lambda \simeq$ $0.1 \mathrm{~nm}$ and $S \simeq 0.1-1 \mathrm{~mm}$, one can estimate $l_{\mathrm{tr}} \simeq 2.5-25 \mu \mathrm{m}$. Since beam coherence over these distances is required to observe the correlations, $l_{\text {tr }}$ sets the ultimate limit of the achievable angular resolution $\delta 2 \theta \simeq \lambda /\left(2 l_{\mathrm{tr}}\right)=S / R$. This resolution can be achieved in the microradian SAXS scheme by exploiting a divergent X-ray beam that is focused at the detector position by CRL installed just in front of the sample (Drakopoulos et al., 2005; Thijssen et al., 2006; Petukhov et al., 2015). One can see that the ultimate angular resolution limit of this scheme is governed by the angular size $S / R$ of the source seen from the position of the sample and is independent of the sample-to-detector distance.

The same conclusion can also be obtained by the following direct-space consideration. Using the geometric optics approach and neglecting the imperfection of the focusing elements, the smallest size of the direct beam at the detector position can be achieved when the lens creates an image of the source at the detector. The demagnification factor of this scheme is governed by the ratio $L / R$ of the lens-detector distance $L$ and the source-lens distance $R$, which defines an ultimate limit for the image size $s=S L / R$. In angular terms, this corresponds to a resolution of $\delta(\theta)=s / L=S / R$.

Thus, by using a convergent X-ray beam that is focused at the detector position, one can readily achieve an angular resolution of the order of 2-20 $\mu \mathrm{rad}$ at a synchrotron source. With the currently ongoing upgrades at the ESRF (The ESRF Orange Book; Dimper et al., 2014) the source size will be reduced even further, which will allow for sub-microradian resolution. This resolution does not necessarily require long beamlines but can also be achieved in a more compact scheme of a few metres long, as long as the detector possesses sufficient resolution (a few micrometres in this case).

\subsection{Novel compact SAXS scheme with tuneable resolution}

The main idea of the proposed novel high-resolution SAXS setup is the use of all the accessible space between the CRL and the fixed position of the $2 \mathrm{D}$ detector as a possible sample 
position, instead of moving the detector relative to a fixed sample. A fixed detector position allows one to use a single detector that is optimized for the resolution of the optical scheme. There is one example of a laboratory SAXS instrument with a movable sample stage and fixed collimation slits and detector position, which allows one to switch easily from wide-angle to small-angle scattering mode (http://www.antonpaar.com/corp-en/products/group/saxs/) but, to the best of our knowledge, such a geometry of SAXS setup with compound refractive lense collimation optics has not been demonstrated yet.

The conceptual scheme of this SAXS setup is presented in Fig. 1. The X-ray beam of the required wavelength passes through the CRL with a short focal distance of the order of 1$2 \mathrm{~m}$. The high-resolution $2 \mathrm{D}$ detector with a small pixel size is placed in the image plane. The sample stage with all the required auxiliary facilities for horizontal, vertical and rotational adjustment of the sample position is mounted on a rail to enable its movement along the beam between the CRL and the detector. The detector stage and the rail with the sample stage can be mounted on an optical table according to the $\mathrm{X}$-ray beam axis and the chosen experimental geometry (see Fig. S1 in the supporting information). Thus, it becomes possible to vary the illuminated area on the sample by moving the sample assembly between the short-focus CRL and the detector along the axis of the converging beam. This allows us to obtain highly averaged information over a large area with high resolution and to zoom in on specific structural features of the sample without the need to realign the optical scheme.

Owing to beam convergence, a change in the sample position relative to the detector and a change in the illuminated region of the sample inevitably lead to a change in the resolution in both direct and reciprocal space. For a change in the transverse coherence length $l_{\text {tr }}$, this will mainly affect the available $q$ range $[q=(4 \pi / \lambda) \sin (\theta / 2)$, where $\theta$ is the scattering angle and $\lambda$ is the wavelength of the incident radiation], depending on the ratio of the detector size and the sample-todetector distance $L_{\text {sd }}$, and also the angular resolution $\Delta q$, depending on the ratio of the pixel size of the detector and $L_{\text {sd }}$. As discussed above, the transverse coherence length $l_{\text {tr }}$, depending on the source size $S$ and distance from the source $R$, will be shrunk, together with the diameter of the irradiated sample area $D$, proportionally to the reduction in the distance

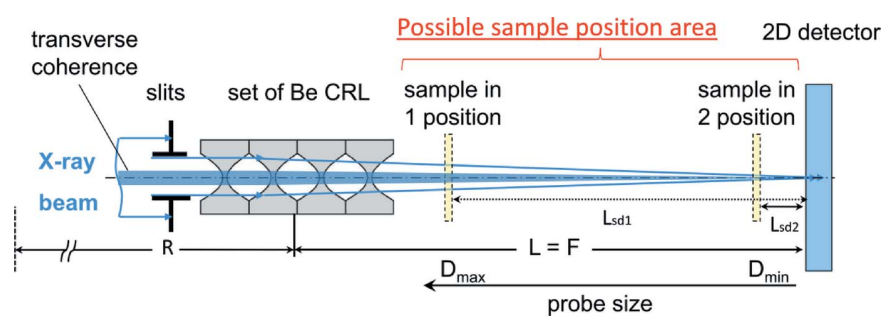

Figure 1

The conceptual scheme of the compact high-resolution small-angle X-ray scattering setup with tuneable probe size. The shaded area of the beam denotes a coherent patch; its size has been exaggerated for better visibility. between the sample and the detector $L_{\mathrm{sd}}$ in the converging $\mathrm{X}$-ray beam. The diameter of the irradiated sample area $D$ during the focusing can be estimated by

$$
D=\left\{\left[\frac{(h-s) L_{\mathrm{sd}}}{F}\right]^{2}+s^{2}\right\}^{1 / 2},
$$

where $h$ is the beam size at the outlet of the CRL, $s$ is the beam size at the detector and $F$ is the focal length of the CRL. The reduction in $l_{\mathrm{tr}}$ will be negligible at the sample position $L_{\text {sd_max }}$ close to the lenses. On the other hand, in a focused converging beam not only its size but also the coherence length $l_{\text {tr }}$ reduce with increasing distance. Owing to the finite value of the coherence length, there is an upper limit on the separation of two objects if they are to give rise to the interference effect. Under the conditions of a small-angle scattering experiment, this corresponds to the period $d$ of the studied nanostructure; hence $L_{\text {sd_min }}$ will depend on the condition $l_{\mathrm{tr}} \geq d$ and, using equations (1) and (2) and neglecting $l_{\mathrm{tr}}$ at the position of the detector, can be estimated approximately as

$$
L_{\mathrm{sd} \_ \text {min }}=\frac{d F}{l_{\mathrm{tr}, 0}}=\frac{2 S d F}{R \lambda} .
$$

Often, the ordered regions of a nano-object have limited dimensions in the sample space and, thus, the sample can contain smaller separate ordered single-crystal regions or domains with various defects (point, linear or volumetric). As the $L_{\mathrm{sd}}$ distance decreases, the size of the irradiated region on the sample also decreases. This makes it possible, in the course of the experiment, to obtain highly averaged information from a significant irradiated sample volume, and to localize on one or several coherent regions of the sample and obtain structural parameters for ordered nano-objects within a particular domain. Note that changing the spatial resolution in direct space is automatically linked to a change in the resolution in reciprocal space.

\section{Experimental}

\subsection{Experimental setup}

The compact high-resolution small-angle X-ray scattering setup with tuneable probe size was assembled on the Microoptics Test Bench installed on beamline ID06 at the European Synchrotron Radiation Facility (ESRF) in Grenoble, France. A schematic drawing of the setup is shown in Fig. 1. Microradian X-ray diffraction with a beam energy of about $12 \mathrm{keV}$ $\left(\lambda=1 \AA, \Delta \lambda / \lambda \simeq 10^{-4}\right)$ was realized using a set of Be CRL, which consisted of 30 parabolic lenses (Snigirev et al., 1996) with a radius of curvature of $200 \mu \mathrm{m}$, giving a focal length $F$ of $1.45 \mathrm{~m}$. The lenses were placed at a distance $R$ of $56 \mathrm{~m}$ from the undulator source, with the source size being $23 \times 928 \mu \mathrm{m}$. The primary slits, closest to the source $(27.8 \mathrm{~m})$, had a vertical gap of $0.5 \mathrm{~mm}$ which did not restrict the beam. In contrast, the horizontal gap of $0.05 \mathrm{~mm}$ was much smaller and effectively defined the secondary source in the horizontal direction at the slit position. Thus in our case, the transverse coherence length at the position of the CRL was about $l_{\mathrm{tr}}=121 \times 28 \mu \mathrm{m}$ in the 
vertical and horizontal directions, respectively. The collimating slits in front of the CRL limited the beam down to $0.5 \mathrm{~mm}$ in the horizontal and vertical directions, thus fitting the effective aperture of the CRL. The beam was focused on a 2D high-resolution Sensicam CCD detector which was combined with an X-ray imaging system. The X-ray imaging system consisted of a $9.9 \mu \mathrm{m}$ thick LAG:Eu scintillator, an Olympus UPLADO tenfold objective and a Sensicam QE camera with a resolution of $1376 \times 1040$ pixels, a pixel size of $6.45 \times 6.45 \mu \mathrm{m}$ and a 12-bit dynamic range. Taking all optical elements into account, the imaging setup enabled an effective pixel size of $0.645 \times 0.645 \mu \mathrm{m}$, covering a field of view of $890 \times 670 \mu \mathrm{m}$. We note that the conditions for best beam focusing are different in the vertical and horizontal directions. The secondary source created in the horizontal direction at the primary beamline slits is located closer to the CRL and its image will be created slightly further downstream. However, the difference in the positions of the images of the primary and secondary sources is small since the focal length $F$ is much smaller than the source-to-lens distance. The currently ongoing upgrade of the ESRF (Dimper et al., 2014) will lead to a nearly circular source with comparable dimensions in the vertical and horizontal directions. In this case, there will no longer be any need to create a secondary source in the horizontal direction, thus removing the problems discussed above that were caused by the very high anisotropy of the source dimensions at the time of the experiment.

The sample holder was installed on a motorized goniometer that allows one to vary the sample-to-detector distance $L_{\mathrm{sd}}$ from 246 to $16 \mathrm{~mm}$. Using slits of $0.5 \times 0.5 \mathrm{~mm}$ or $0.2 \times 0.2 \mathrm{~mm}$, this range of $L_{\mathrm{sd}}$ corresponds to illuminated area sizes from $87 \times 87 \mu \mathrm{m}$ to $7.5 \times 7.5 \mu \mathrm{m}$ and from $35.6 \times 35.6 \mu \mathrm{m}$ to $4.2 \times 4.2 \mu \mathrm{m}$, respectively. As a test sample we used a $96 \mu \mathrm{m}$ thick anodic alumina membrane with a selfordered porous structure (a pore diameter of $c a 40 \mathrm{~nm}$, with the distance between the centres of neighbouring pores $d_{\text {int }}$ being $102 \mathrm{~nm}$ ). The membrane was installed perpendicular to the incident beam on the translation/rotation stage with possible translation along the beam axis. Using the minimum illuminated area size of $4.2 \mu \mathrm{m}$, focusing on the area of one or a few structure domains became possible. Here we present data for a slit size of $0.2 \times 0.2 \mathrm{~mm}$ with varying diameters of the irradiated area $D=35.6,31.5,24.7,17.8,11$ and $4.2 \mu \mathrm{m}$ at sample-to-detector distances $L_{\mathrm{sd}}=246,216,166,116,66$ and $16 \mathrm{~mm}$, respectively. The minimum sample-to-detector distance $L_{\text {sd_min }}$ for our case at $l_{\mathrm{tr}}=d$, according to equation (3), should be about $2.5-5 \mathrm{~mm}$.

Scanning electron microscopy (SEM) images were recorded using an NVision 40 scanning electron microscope (Carl Zeiss). To examine the in-plane orientational order in the AAO structure, statistical analysis of the SEM images was performed using a colour-coding procedure (Hillebrand et al., 2008). The statistical distribution of the interpore distances for the nearest neighbours around the considered pore was obtained by the Voronoi algorithm using the ImageJ program (Schneider et al., 2012) and in-house software (http:// eng.fnm.msu.ru/en/software/).

\subsection{Sample description}

AAO is a typical self-ordered nanostructured material with a two-dimensional hexagonal network of pores (Masuda \& Fukuda, 1995). Since that pioneering work, which showed the opportunity for the formation of large-scale ordered structures, AAO has been intensively used as a platform for creating various nanostructured functional devices (Qin et al., 2005; McGary et al., 2006; Masuda et al., 2006; Banerjee et al., 2009; Kolmychek et al., 2018). The real structure of a selfordered AAO membrane consists of domains of longitudinal pores. In each domain, the pore structure seeks to be ordered. The pores can branch and terminate during growth of the membrane, and these processes occur mainly at the domain boundaries (Napolskii et al., 2011). Defects are inherent in the real structure of anodic alumina and they have an influence on both the diffraction patterns and the functional properties of AAO membranes. Napolskii et al. (2010) and Roslyakov et al. (2016) demonstrated that microradian X-ray diffraction is an efficient tool for the structural characterization of self-ordered porous oxide films. In contrast with conventional microscopy techniques, the SAXS technique allows one to determine quantitatively the $3 \mathrm{D}$ ordering of self-organized AAO structures.

The anodic alumina membranes were prepared using the two-stage anodizing technique. High-purity aluminium foil (99.999\%, $0.5 \mathrm{~mm}$ thick, Goodfellow) with a coarse grain structure was used as the starting material. The lateral sizes of the aluminium grains were higher than $1 \mathrm{~mm}$, and their thickness coincided with the foil thickness. Prior to anodizing, the foil was mechanically polished using a diamond suspension (Struers) to a mirror finish and cleaned repeatedly with acetone and deionized water in an ultrasonic bath. Then the aluminium was anodized in $0.3 \mathrm{M}$ oxalic acid $\left(\mathrm{H}_{2} \mathrm{C}_{2} \mathrm{O}_{4}, 98 \%\right.$, Aldrich) at $40 \mathrm{~V}$ in a two-electrode cell using $\mathrm{Pt}$ wire as the counter electrode. The temperature of the electrolyte was kept in the range of $273-274 \mathrm{~K}$. These conditions lead to the formation of oxide films with a highly ordered porous structure, with the domains possessing a nearly ideal $2 \mathrm{D}$ hexagonal pore arrangement (Roslyakov et al., 2017). In the plane of the oxide film the domains have an irregular shape and their maximum lateral size can reach several or even a few tens of micrometres. After the first stage of anodizing for $72 \mathrm{~h}$, the alumina film was selectively etched away in an aqueous solution containing $35 \mathrm{ml} \mathrm{l}^{-1} 85 \% \mathrm{H}_{3} \mathrm{PO}_{4}$ and $20 \mathrm{~g} \mathrm{l}^{-1} \mathrm{CrO}_{3}$ at $343 \mathrm{~K}$. The second anodizing stage was performed under the same conditions for $47 \mathrm{~h}$. The thickness of the obtained AAO film was about $96 \mu \mathrm{m}$.

\section{Results and discussion}

Anodic alumina membranes possess a significant scattering contrast, caused by the difference between the refractive indices of air in the longitudinal pores and of the material of the pore walls. We used AAO films with a self-ordered porous structure (the distance between the centres of the pores $d_{\text {int }}=$ $102 \mathrm{~nm}$ ), consisting of domains (regions with 2D hexagonal 


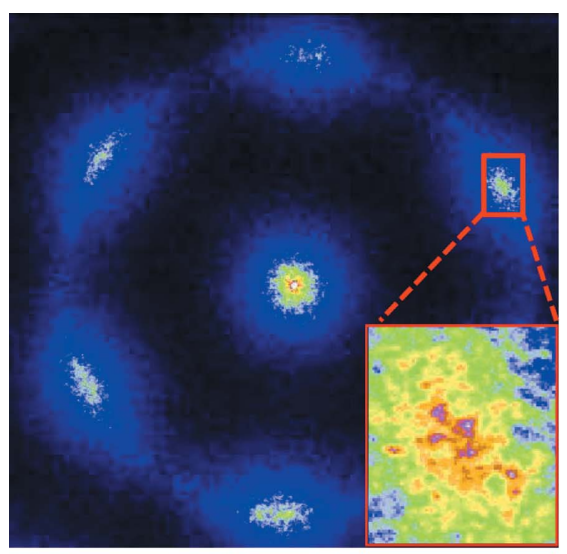

(a)

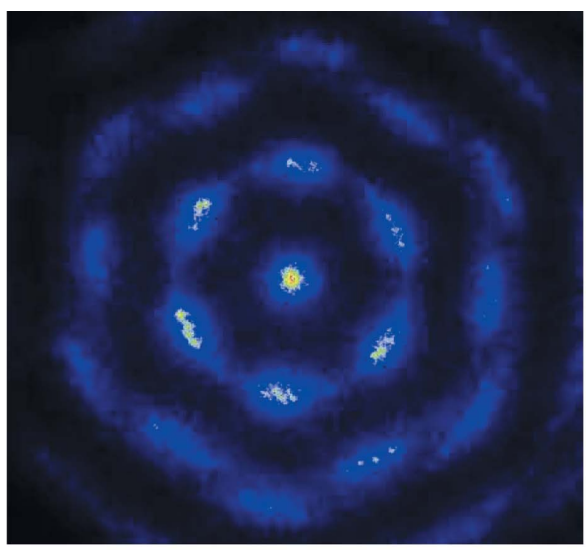

(b)

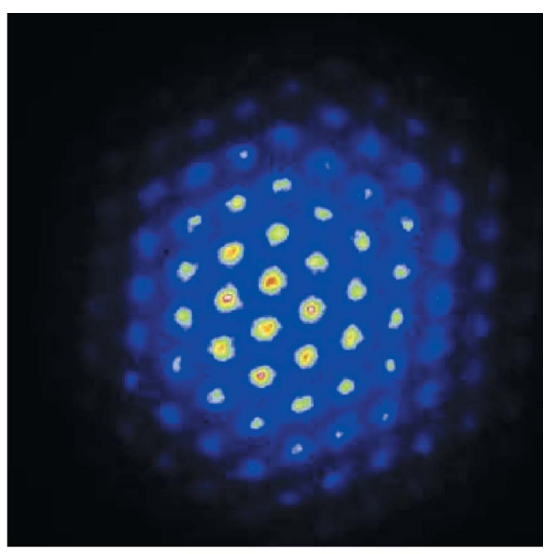

(c)

Figure 2

2D X-ray diffraction patterns of porous anodic alumina obtained at different sample-to-detector distances $L_{\mathrm{sd}}$ and irradiated spot diameters $D$. $(a) L_{\mathrm{sd}}=$ $246 \mathrm{~mm}, D=35.6 \mu \mathrm{m}$. (b) $L_{\mathrm{sd}}=116 \mathrm{~mm}, D=17.8 \mu \mathrm{m}$. (c) $L_{\mathrm{sd}}=16 \mathrm{~mm}, D=4.2 \mu \mathrm{m}$.

pore ordering) whose average size is 1$10 \mu \mathrm{m}$. This range of sizes of 'ideally' ordered regions allows us to realize a situation where the length of the transverse coherence of the beam is larger, comparable to or less than the size of the domains.

$2 \mathrm{D}$ diffraction patterns of the AAO membrane, recorded at different sample-to-detector distances $L_{\mathrm{sd}}=246$, 116 and $16 \mathrm{~mm}$, are shown in Fig. 2. The SAXS data show that some scattering intensity is present in almost all azimuthal directions, which is in good agreement with the powder-like domain structure of AAO revealed by SEM [Figs. 3(b) and 3(c)]. However, a clear sixfold modulation of the scattered intensity suggests that, on a large scale, a predominant range of orientations prevails. Earlier, we performed a detailed statistical analysis of the SEM data (Napolskii et al., 2012), which confirmed that a seemingly powder-like domain structure of a single SEM image reveals a predominant orientation after averaging over many neighbouring spots.

The sizes of the transverse coherence will change during beam focusing. On the basis of the parameters calculated above for the vertical and horizontal transverse coherence of the beam at the position of the CRL and assuming that the coherence length $l_{\text {tr }}$ reduces linearly with increasing distance in a convergent beam, $l_{\mathrm{tr}}=l_{\mathrm{tr}, 0} L_{\mathrm{sd}} / F$, we calculate the following values of the transverse coherence length for all
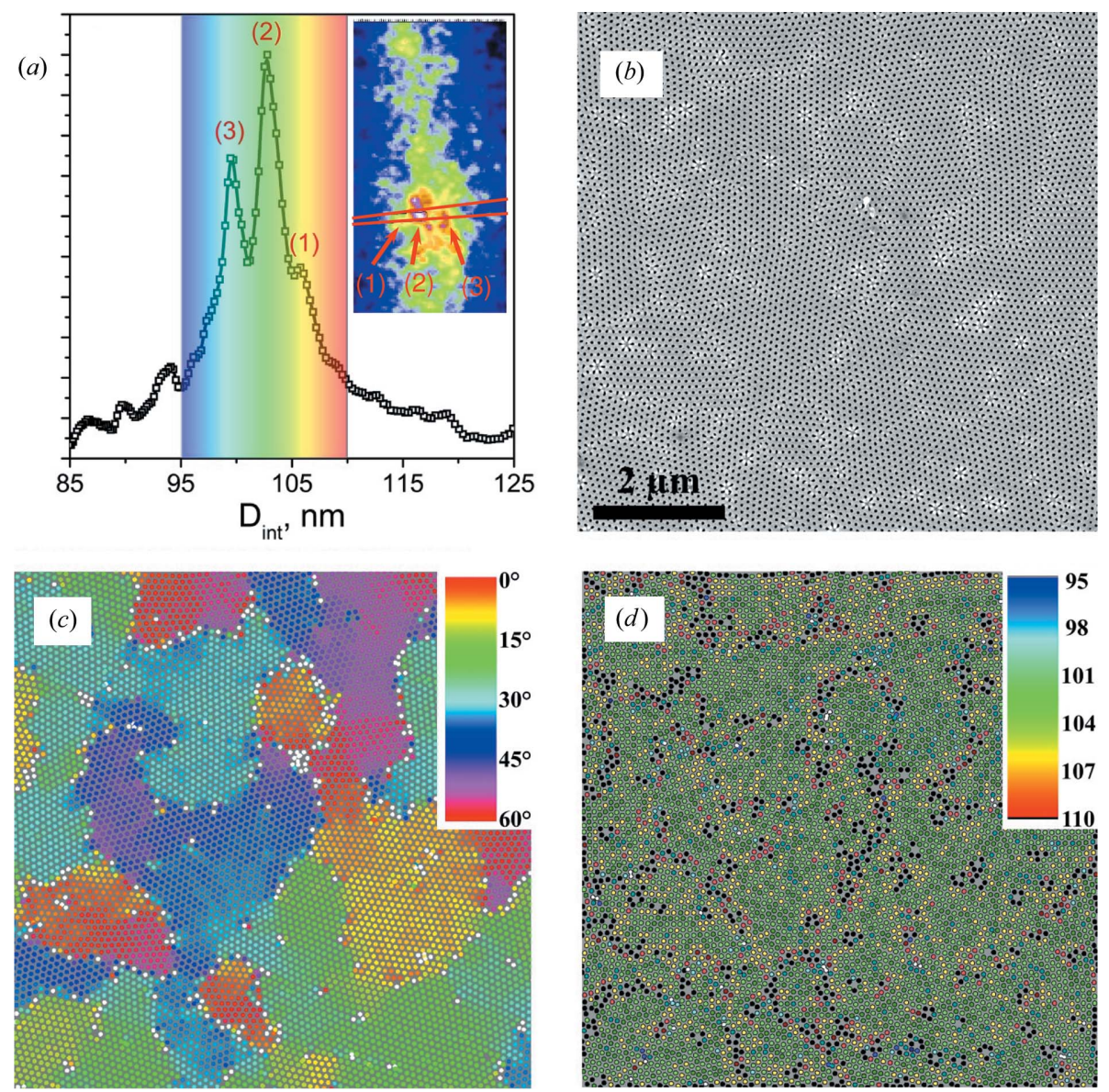

Figure 3

(a) The pair distribution function calculated from the momentum-transfer dependence of the X-ray scattering for a narrow sector with an opening of $\delta \varphi=0.6^{\circ}$ for the first-order diffraction peak (see inset). The diffraction pattern was recorded at a sample-to-detector distance $L_{\mathrm{sd}}$ of $216 \mathrm{~mm}$. The strong point Bragg reflections are marked by numerals (1), (2) and (3). (b) A SEM image of the bottom side of AAO (after removal of the barrier layer). (c), (d) The SEM image from panel $(b)$, subjected to the colour coding procedure. $(c)$ The colour indicates the average angle to the nearest neighbours of the considered pore. $(d)$ The colour shows the average interpore distance to the nearest neighbours. The corresponding colour scales in degrees and nanometres are given in the upper right corners of corresponding panels. 
three positions of Fig. 2: (a) $20.53 \times 0.40 \mu \mathrm{m}, \quad$ (b) $9.68 \times 0.19 \mu \mathrm{m}$ and $(c) 1.34 \times 0.03 \mu \mathrm{m}$ in the vertical and horizontal directions, respectively. One can see that the number of high-order reflections increases when the sample is closer to the detector. Moreover, they shift closer to the position of the directly transmitted beam. It was revealed that the diffraction peaks consist of aggregates of small point diffraction reflections which are the result of X-ray scattering on sets of pores with different interpore distances [see the inset in Fig. 2(a)]. Pore arrays with a longitudinal axis direction coaxial with the X-ray beam produce brighter point diffraction reflections than other domains with disorientation of the pores relative to the incident beam. It should be noted that the point diffraction reflections are not speckles of the X-ray beam because the brightest ones repeat every $60^{\circ}$, following the hexagonal symmetry of the AAO structure. It was also possible to observe the appearance and extinction of a single point diffraction reflection in a rocking curve experiment (Fig. S2 in the supporting information).

The obtained 2D patterns of small-angle diffraction can be analysed on the basis of the previously demonstrated technique for determining the parameters of the AAO structure by the SAXS method (Napolskii et al., 2010). Let us examine the momentum transfer $q$ dependence of the scattering intensity for a narrow sector with an opening of $\delta \varphi=0.6^{\circ}$ for the firstorder diffraction peak [see the inset in Fig. 3(a)]. It can be seen that the first-order diffraction maximum, which is usually smooth (Napolskii et al., 2010), splits into three independent and clearly distinguishable diffraction peaks [marked (1), (2) and (3)]. The dependencies $I(q)$ were fitted by the sum of Lorentz functions. The point-like diffraction peaks each have unique locations with centres at (1) $q_{10}=0.06844$ (4) $\mathrm{nm}^{-1}$, (2) $q_{10}=0.07052$ (1) $\mathrm{nm}^{-1}$ and (3) $q_{10}=0.07268$ (1) $\mathrm{nm}^{-1}$. These values of the momentum transfer $q$ correspond to structures with the following interpore distances: (1) $d_{\text {int_1 } 1}=106.0 \mathrm{~nm}$, (2) $d_{\text {int_2 }}=102.9 \mathrm{~nm}$ and (3) $d_{\text {int_3 }}=99.8 \mathrm{~nm}$ [see the pair distribution function in Fig. 3(a)]. Note that the average interpore distance calculated from the radial intensity profile $I(q)$ averaged over the full azimuthal range $\left(\delta \varphi=360^{\circ}\right)$ gives us a value of $d_{\text {int }}=101.4 \mathrm{~nm}$.

There are two possible reasons for the existence of separate maxima on the pair distribution function corresponding to areas with various interpore distances: (i) the AAO porous structure consists of domains in which the average interpore distance varies from domain to domain; (ii) areas with much lower or higher distances between nearest-neighbour pores are localized near defect regions, where the pore surroundings are far from hexagonal. According to SEM images of the bottom side of AAO porous films [Fig. 3(b)], the second scenario is realized. Colour maps of the domain in-plane orientation and interpore distance distributions are shown in Figs. 3(c) and 3(d), respectively. One can see that the average interpore distance inside domains with various in-plane orientations does not vary significantly. As a consequence, the multimodal nature of the radial SAXS profiles and of the corresponding pair distribution functions with sharp satellites arises from defect areas of the porous structure, which are marked as white and black points in the colour-coded SEM images. Thus, it can be concluded that the bright point diffraction reflections arise from the ordered pore structure within the domains, whereas the defect areas of the porous film located near domain boundaries and point defects are the sources of the small maxima on the overall background of the diffraction peak.

Defining the positional correlations of the pores as $m=q_{10} / \delta q_{10}$ from the radial width $\delta q_{10}$ of the lowest-order Bragg peak, we find that for the point diffraction reflections the value $m$ varies from 30 to 80 structure periods in each domain. In the case of full-circle averaging, the $m$ value varies in the range of 5-7 structure periods. Such a decrease in the number of average structure periods in ordered regions with increasing irradiated area is in good agreement with the assumption that pore branching and termination occur mainly on the boundaries of the domains (Napolskii et al., 2011).

The azimuthal dependence of the first-order Bragg reflection intensity $I(\varphi)$ calculated from diffraction patterns recorded at different diameters of illuminated area $D$ from 35.6 to $4.2 \mu \mathrm{m}$ is shown in Fig. 4. As the irradiated area decreases, the evolution of the point diffraction reflections and their contribution to the overall scattering intensity $I(\varphi)$ of the diffraction peak can be observed. We can conclude that a significant part of the domains has an advantageously unidirectional orientation of the pore lines in the plane of the membrane, which is governed by the crystallographic orientation of the aluminium substrate (Napolskii et al., 2012). It can be clearly seen that a decrease in the sample-to-detector

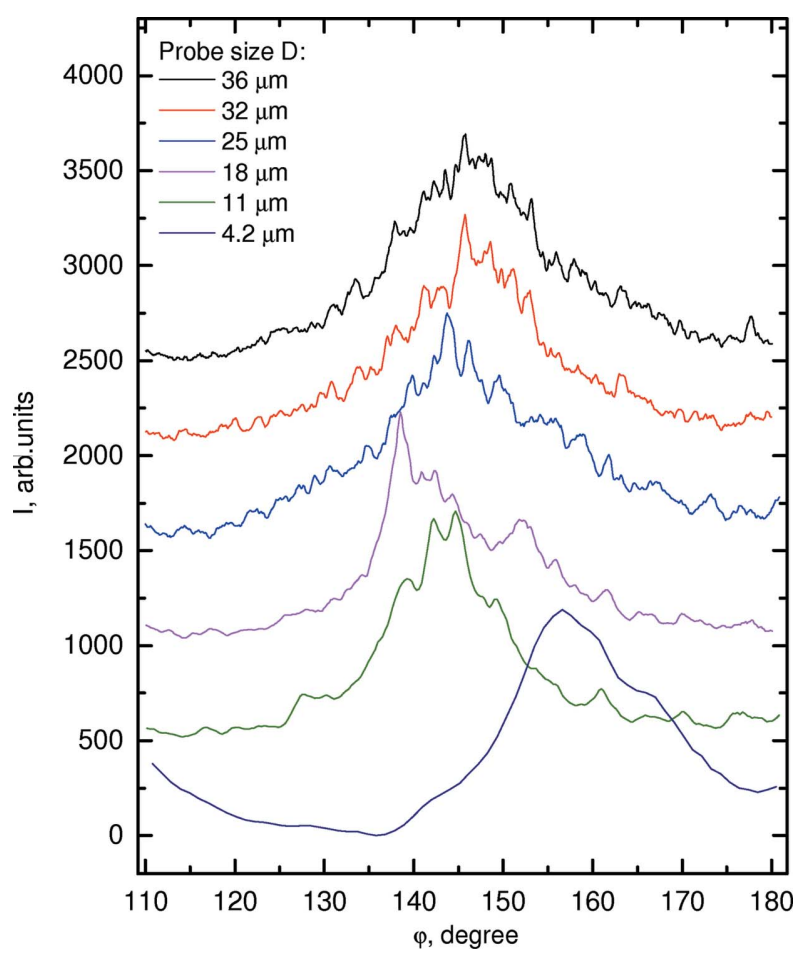

Figure 4

The azimuthal intensity distribution $I\left(\varphi_{10}\right)$ of the 10 diffraction maximum $\left(110<\varphi<180^{\circ}\right)$ at irradiated spot diameters $D$ from 36 to $4.2 \mu \mathrm{m}$ (at sample-to-detector distances $L_{\text {sd }}$ from 246 to $16 \mathrm{~mm}$ ). 
distance leads to a lesssening of the resolution in reciprocal space and as a consequence the $I(\varphi)$ curves become smooth.

As we have demonstrated in our previous work (Napolskii et al., 2010; Grigoriev et al., 2010; Chumakov et al., 2013; Roslyakov et al., 2013), diffraction patterns are extremely sensitive to sample orientation. Rotation of the sample by as little as $0.1^{\circ}$ around the axes orthogonal to the beam leads to a significant modification of the diffraction pattern. The width of the rocking curve $\delta \omega$ is related to the correlation length of the porous structure along with the pores $\left(L_{z}\right)$ via the equation

$$
L_{z}=\frac{2 \pi}{\delta q_{z}}=\frac{2 \pi}{q_{10} \sin \delta \omega},
$$

where $\delta q_{z}$ is the width of the 10 diffraction maximum along the beam, $q_{10}$ is the scattering vector corresponding to the 10 diffraction peak and $\delta \omega$ is the FWHM of the rocking curve. We can define the longitudinal self-correlation length as the length along the pore within which the difference between the pore and a best-fit straight cylinder is smaller than the pore diameter.

Fig. 5 presents the rocking curves for the intensity of the 10 diffraction peak at sample-to-detector distances of $(a) L_{\mathrm{sd}}=$ $216 \mathrm{~mm}$ and $(b) L_{\mathrm{sd}}=16 \mathrm{~mm}$, with the diameters of the irradiated areas being $D=31.5$ and $4.2 \mu \mathrm{m}$, respectively. Each point on the experimental curve represents the integral

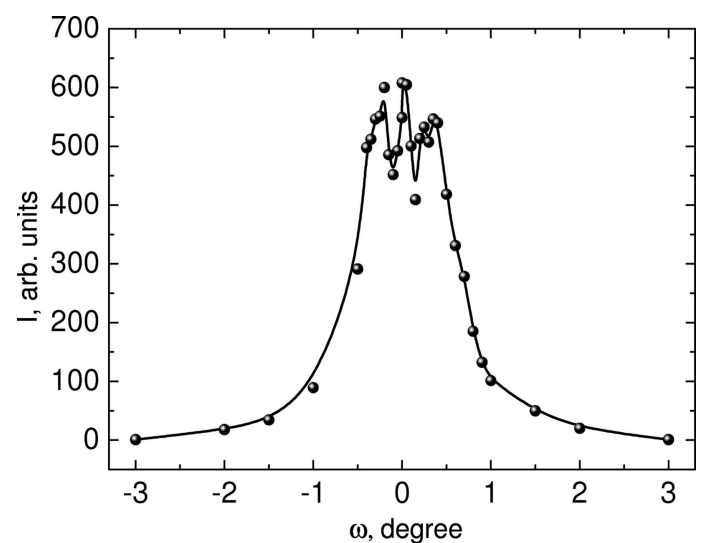

(a)

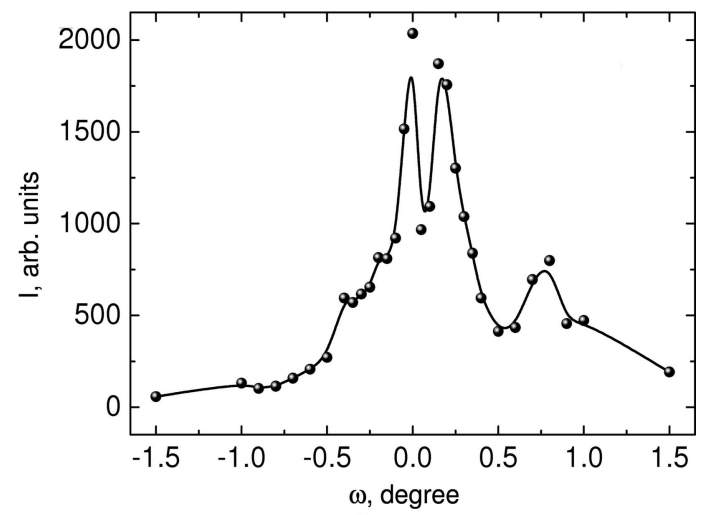

(b)

Figure 5

Rocking curves of the first-order peak $q_{10}$ with the azimuthal peak centre $\varphi=0^{\circ}$ and opening $\delta \varphi=14^{\circ}$ at sample-to-detector distances $(a) L=$ $216 \mathrm{~mm}$ and $(b) L=16 \mathrm{~mm}$. intensity of a diffraction peak obtained from the Lorentzian fit of the radial distribution of the scattered intensity $I(q)$ in the azimuthal range $\delta \varphi=14^{\circ}$. The FWHM of the rocking curve shown in Fig. $5(a)$ is $\delta \omega=1.366^{\circ}$, which corresponds to a correlation length of $L_{z} \simeq 3.7 \mu \mathrm{m}$. In the case of the small spot of irradiated area $D=4.2 \mu \mathrm{m}$, the rocking curve is formed by a set of clearly visible peaks [Fig. 5(b)]. Taking into account the presence of just a few domains within the small irradiated area, we can explain the narrow peaks as rocking curves from the different point diffraction reflections. The $\delta \omega$ values for these peaks are about $0.05^{\circ}$ and correspond to a correlation length $L_{z} \simeq 100 \mu \mathrm{m}$, which is very close to the membrane thickness $(96 \mu \mathrm{m})$. Thus, we can conclude that the real longitudinal self-correlation length (the straight section of the pores) inside the domain significantly exceeds the average longitudinal correlation length of the membrane. The pores within the domains are mainly rectilinear through the whole membrane thickness.

Moving the sample closer to the detector results in focusing of the X-ray beam on the local volume of the specimen, which allows us to study the local features of the AAO structure. Using an X-ray beam with a minimal beam size of $D=4.2 \mu \mathrm{m}$ enables focusing on the area of a unit domain or on the boundary between two domains to choose the most appropriate region of interest (Fig. 6). Using a sample-to-detector distance of $16 \mathrm{~mm}$, we performed a mapping of the structure
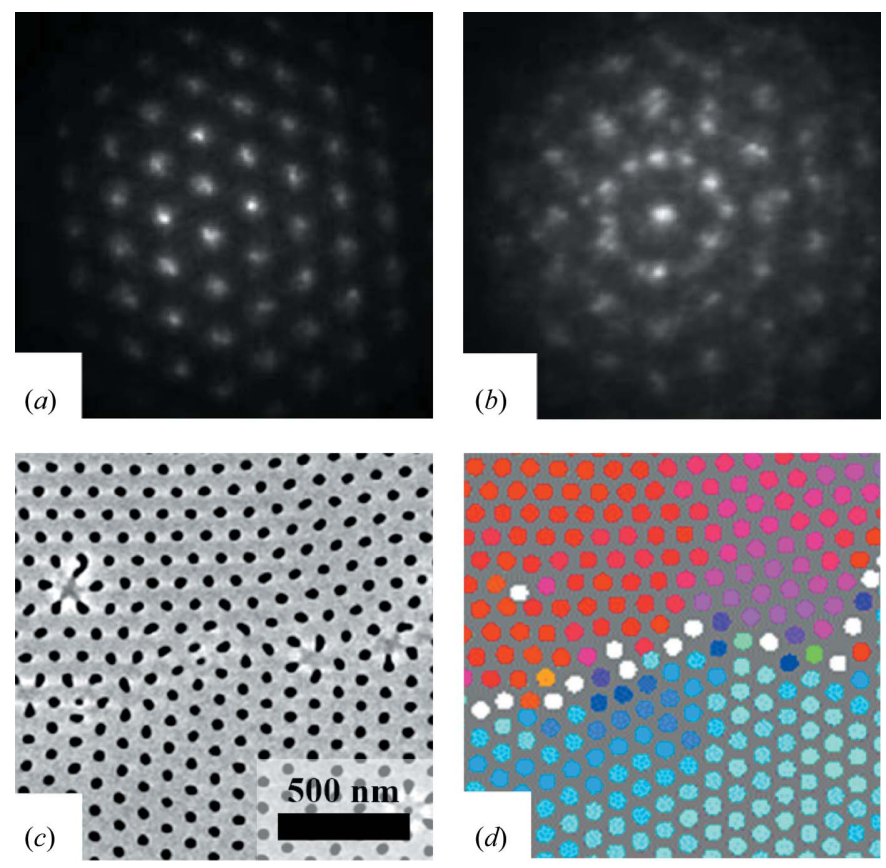

Figure 6

Diffraction patterns recorded at two points on the AAO sample situated at a distance of $3 \mu \mathrm{m}$ apart. The X-ray beam passes through $(a)$ the centre of the domain and $(b)$ the region of the boundary of two domains. The sample-to-detector distance $L_{\mathrm{sd}}$ is $16 \mathrm{~mm}(D=4.2 \mu \mathrm{m})$. (c) A SEM image of AAO in a randomly chosen region of the high-angle border between two neighbouring domains. (d) The SEM image from panel (c) subjected to colour coding based on the average angle to the nearest neighbours of the considered pore [the colour scale is the same as in Fig. 3(c)]. 
with a resolution equal to the minimum movement of the stepper motors.

It can be clearly seen that the mosaicity of the porous structure inside the domain [Fig. $6(a)$ ] is very low $\left(\delta \varphi \simeq 15^{\circ}\right)$. As a consequence, the average mosaicity of the AAO structure is mainly given by the disorientation of neighbouring domains rather than by the variation of the in-plane orientation of the pore structure inside the domain. The diffraction pattern recorded from the region of the domain boundary [Fig. 6(b)] corresponds to the high-angle boundary: the six first-order reflections from the first domain are rotated by $\varphi \simeq$ $30^{\circ}$ with respect to the six reflections from the neighbouring domain. Such $30^{\circ}$ boundaries are common for the AAO porous structure. As an example, a high-magnification SEM image of a randomly chosen region of the AAO structure near the domain boundary is shown in Fig. 6(c). The corresponding colour-coded map [Fig. $6(\mathrm{~d})$ ] showing the $30^{\circ}$ disorientation between two domains is in good agreement with the diffraction pattern with 12 -fold intensity modulation [Fig. 6(b)].

\section{Conclusions}

We have successfully demonstrated the advantages of the proposed novel SAXS scheme:

(i) Compact SAXS with a tuneable probe size is well suited to the investigation of both the average and local structure of nanomaterials with spatially ordered structure.

(ii) The use of CRL allows one to reduce the length of the SAXS setup to $c a 1.5 \mathrm{~m}$.

(iii) Changing the slit gap behind the CRL, and the sample position between the CRL and the detector, allows one to change the irradiated area on the sample.

(iv) Diffraction mapping of the sample is possible at a minimal sample-to-detector distance.

(v) Scattering in air is reduced in a compact setup with a shorter lens-to-detector distance, so it is no longer necessary to use a vacuum detector tube for samples with sufficient contrast.

(vi) It would be possible to construct this easy scheme as a temporary assembly at different laboratories and synchrotron beamlines.

The proposed SAXS scheme should also be examined for its possible drawbacks:

(i) The resolution parameters in reciprocal space begin to deteriorate during the approach of the sample to the detector, owing to the shrinkage of reciprocal space in the detector plane.

(ii) To achieve sufficient resolution in reciprocal space in the compact SAXS scheme, a high-resolution detector with minimal pixel size is required.

Summing up our test investigation of AAO membranes by compact SAXS with a tuneable probe size, we make the following observations:

(i) The interpore distance distribution is narrow. Areas with much shorter or longer distances between nearest-neighbour pores are localized near defect regions, where the pore surroundings are far from hexagonal. (ii) Each domain of the AAO membrane consists of rectilinear through pores. The longitudinal pore axes between the domains have a different reorientation on an angle of about $0.05-0.5^{\circ}$ from domain to domain.

(iii) The dispersion of point reflections into diffraction peaks testifies to the reorientation of domains in the plane of the membrane.

(iv) The pore structure inside the domains is close to the ideal 2D hexagonal structure. At the high-angle domain boundaries the misorientation of the nearest domains is close to $30^{\circ}$, but low-angle boundaries lead to a smooth change of the in-plane orientation of the porous system from one domain to another.

\section{Acknowledgements}

The authors acknowledge the ESRF for hospitality and Yuriy Chushkin (XPCS/CXDI, ID10 beamline, ESRF) for fruitful discussions.

\section{Funding information}

Synthesis and SEM characterization of the anodic alumina films were performed with the financial support of the Russian Science Foundation (grant No. 18-73-10151). The development of the high-resolution small-angle $X$-ray scattering setup was supported by RSF grant No. 19-72-30009. AAS is grateful to the Russian Academic Excellence Project at the Immanuel Kant Baltic Federal University for financial support of his travel.

\section{References}

Als-Nielsen, J. \& McMorrow, D. (2011). Elements of Modern X-ray Physics. Chichester: John Wiley \& Sons.

Banerjee, P., Perez, I., Henn-Lecordier, L., Lee, S. B. \& Rubloff, G. W. (2009). Nat. Nanotechnol. 4, 292-296.

Born, M. \& Wolf, E. (1970). Principles of Optics, 4th ed. Oxford: Pergamon Press.

Bosak, A., Snigireva, I., Napolskii, K. \& Snigirev, A. (2010). Adv. Mater. 22, 3256-3259.

Byelov, D. V., Meijer, J.-M., Snigireva, I., Snigirev, A., Rossi, L., van den Pol, E., Kuijk, A., Philipse, A., Imhof, A., van Blaaderen, A., Vroege, G. J. \& Petukhov, A. V. (2013). RSC Adv. 3, 15670-15677.

Chumakov, A. P., Roslyakov, I. V., Napolskii, K. S., Eliseev, A. A., Lukashin, A. V., Eckerlebe, H., Bouwman, W. G., Belov, D. V., Okorokov, A. I. \& Grigoriev, S. V. (2013). Nanotechnol. Russia, 8, 631-638.

Dimper, R., Reichert, H., Raimondi, P., Sánchez Ortiz, L., Sette, F. \& Susini, J. (2014). The ESRF Orange Book: ESRF Upgrade Programme Phase II (2015-2022) Technical Design Study. ESRF, Grenoble, France. http://www.esrf.eu/Apache_files/Upgrade/ESRForange-book.pdf.

Drakopoulos, M., Snigirev, A., Snigireva, I. \& Schilling, J. (2005). Appl. Phys. Lett. 86, 014102.

Grigoriev, S. V., Syromyatnikov, A. V., Chumakov, A. P., Grigoryeva, N. A., Napolskii, K. S., Roslyakov, I. V., Eliseev, A. A., Petukhov, A. V. \& Eckerlebe, H. (2010). Phys. Rev. B, 81, 125405.

Hillebrand, R., Müller, F., Schwirn, K., Lee, W. \& Steinhart, M. (2008). ACS Nano, 2, 913-920. 
Ilavsky, J., Jemian, P. R., Allen, A. J., Zhang, F., Levine, L. E. \& Long, G. G. (2009). J. Appl. Cryst. 42, 469-479.

Kolmychek, I. A., Pomozov, A. R., Leontiev, A. P., Napolskii, K. S. \& Murzina, T. V. (2018). Opt. Lett. 43, 3917-3920.

Lengeler, B., Schroer, C. G., Kuhlmann, M., Benner, B., Günzler, T. F., Kurapova, O., Zontone, F., Snigirev, A. \& Snigireva, I. (2005). J. Phys. D Appl. Phys. 38, A218-A222.

Masuda, H. \& Fukuda, K. (1995). Science, 268, 1466-1468.

Masuda, H., Yamada, M., Matsumoto, F., Yokoyama, S., Mashiko, S., Nakao, M. \& Nishio, K. (2006). Adv. Mater. 18, 213-216.

McGary, P. D., Tan, L. W., Zou, J., Stadler, B. J. H., Downey, P. R. \& Flatau, A. B. J. (2006). Appl. Phys. 99, 08 B310.

Napolskii, K., Roslyakov, I., Romanchuk, A., Kapitanova, O., Mankevich, A., Lebedev, V. \& Eliseev, A. (2012). J. Mater. Chem. 22, 11922-11926.

Napolskii, K. S., Roslyakov, I. V., Eliseev, A. A., Byelov, D. V., Petukhov, A. V., Grigoryeva, N. A., Bouwman, W. G., Lukashin, A. V., Chumakov, A. P. \& Grigoriev, S. V. (2011). J. Phys. Chem. C, 115, 23726-23731.

Napolskii, K. S., Roslyakov, I. V., Eliseev, A. A., Petukhov, A. V., Byelov, D. V., Grigoryeva, N. A., Bouwman, W. G., Lukashin, A. V., Kvashnina, K. O., Chumakov, A. P. \& Grigoriev, S. V. (2010). J. Appl. Cryst. 43, 531-538.

Narayanan, T., Sztucki, M., Van Vaerenbergh, P., Léonardon, J., Gorini, J., Claustre, L., Sever, F., Morse, J. \& Boesecke, P. (2018). J. Appl. Cryst. 51, 1511-1524.

Nikitenko, S., Beale, A. M., van der Eerden, A. M. J., Jacques, S. D. M., Leynaud, O., O’Brien, M. G., Detollenaere, D., Kaptein, R., Weckhuysen, B. M. \& Bras, W. (2008). J. Synchrotron Rad. 15, 632640.

Pauw, B. R. (2013). J. Phys. Condens. Matter, 25, 383201.

Petukhov, A. V., Aarts, D. G. A. L., Dolbnya, I. P., de Hoog, E. H. A., Kassapidou, K., Vroege, G. J., Bras, W. \& Lekkerkerker, H. N. W. (2002). Phys. Rev. Lett. 88, 208301.

Petukhov, A. V., Meijer, J.-M. \& Vroege, G. J. (2015). Curr. Opin. Colloid Interface Sci. 20, 272-281.
Petukhov, A. V., Thijssen, J. H. J., 't Hart, D. C., Imhof, A., van Blaaderen, A., Dolbnya, I. P., Snigirev, A., Moussaï, A. \& Snigireva, I. (2006). J. Appl. Cryst. 39, 137-144.

Qin, L. D., Park, S., Huang, L. \& Mirkin, C. A. (2005). Science, 309, 113-115.

Riekel, C., Burghammer, M. \& Davies, R. (2010). IOP Conf. Ser. Mater. Sci. Eng. 14, 012013.

Roslyakov, I. V., Eliseev, A. A., Yakovenko, E. V., Zabelin, A. V. \& Napolskii, K. S. (2013). J. Appl. Cryst. 46, 1705-1710.

Roslyakov, I. V., Gordeeva, E. O. \& Napolskii, K. S. (2017). Electrochim. Acta, 241, 362-369.

Roslyakov, I. V., Koshkodaev, D. S., Eliseev, A. A., Hermida-Merino, D., Petukhov, A. V. \& Napolskii, K. S. (2016). J. Phys. Chem. C, 120, 19698-19704.

Santoro, G., Buffet, A., Döhrmann, R., Yu, S., Körstgens, V., MüllerBuschbaum, P., Gedde, U., Hedenqvist, M. \& Roth, S. V. (2014). Rev. Sci. Instrum. 85, 043901.

Schneider, C. A., Rasband, W. S. \& Eliceiri, K. W. (2012). Nat. Methods, 9, 671-675.

Seiboth, F., Schropp, A., Hoppe, R., Meier, V., Patommel, J., Lee, H. J., Nagler, B., Galtier, E. C., Arnold, B., Zastrau, U., Hastings, J. B., Nilsson, D., Uhlén, F., Vogt, U., Hertz, H. M. \& Schroer, C. G. (2014). J. Phys. Conf. Ser. 499, 012004.

Smith, A. J., Davidson, L. S., Emmins, J. H., Hiller Bardsley, J. C., Holloway, P., Malfois, M., Marshall, A. R., Pizzey, C. L., Rogers, S. E., Shebanova, O., Snow, T., Williams, E. P. \& Terrill, N. J. (2019). arXiv:1903.05405.

Snigirev, A., Kohn, V., Snigireva, I. \& Lengeler, B. (1996). Nature, 384, 49-51.

Snigireva, I. \& Snigirev, A. (2006). J. Environ. Monit. 8, 33-42.

Thijssen, J. H. J., Petukhov, A. V., 't Hart, D. C., Imhof, A., van der Werf, C. H. M., Schropp, R. E. I. \& van Blaaderen, A. (2006). $A d v$. Mater. 18, 1662-1666.

Vaughan, G. B. M., Wright, J. P., Bytchkov, A., Rossat, M., Gleyzolle, H., Snigireva, I. \& Snigirev, A. (2011). J. Synchrotron Rad. 18, 125133. 